\title{
Detection and localization of slag inclusions in weld using IR thermography
}

\author{
by W. Jamrozik and J. Górka
}

\author{
Sielsian Univ. of Technology, 44-100, 2A Akademicka str., Gliwice, Poland, wojciech.jamrozik@polsl.pl
}

\begin{abstract}
Slag inclusions are common welding imperfections, decreasing quality of joint. In the paper an on-line method of slag detection and localization is proposed for manual electrode welding. Two perpendicularly placed IR cameras are used to observe welding. Based on topological analysis of welding arc area and dynamics of weld cooling defects are found and localized in seam. To overcome errors in emissivity setting, relative temperature change were used. Results were confirmed with radiographic examination of joints. It was found that proposed method can be successfully used for slag detection of certain size. Minimal size of detected defects has to be determined in further studies.
\end{abstract}

\section{Introduction}

The phenomenon of slag inclusion is a one of common imperfection (Fig. 1) that can occur when joining metallic materials using welding technologies. It is in general a product of reaction between the flux, air and surface oxide. Since the melting temperatures of surface oxides are usually much higher than the base metal, they are more likely to stay solid during welding and become trapped in the weld pool. During TIG welding, if the tungsten electrode accidentally contacts the molten weld pool or if there is too high weld current, tungsten inclusions can be produced in the join area. Elements with a strong affinity for oxygen, such as aluminium or magnesium, can relatively easy react with oxygen to form oxide inclusions in the weld metal. Other reasons that lead to fact that slag is maintained in the welded seam are connected with technological as well as operational errors. Slag can be kept in the weld surface when welding current density is to small to heat enough the molten metal or the welding pool is cooled to fast. Moreover when the welding speed is to high slag can not be always float from welding area. Operator errors can also increase the possibility of slag inclusion, e.g. when in multi-pass welding slag layer is not properly removed before or the material edges are not completely clean before making consecutive seams. In other words, when deposing next layer, the entrapped slag is not melted out.

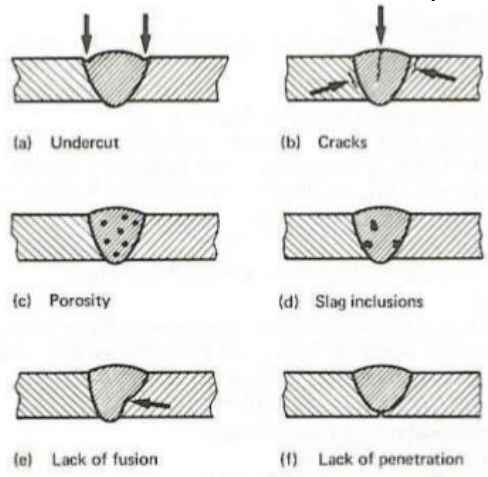

Fig. 1. Typical weld imperfections (defects)

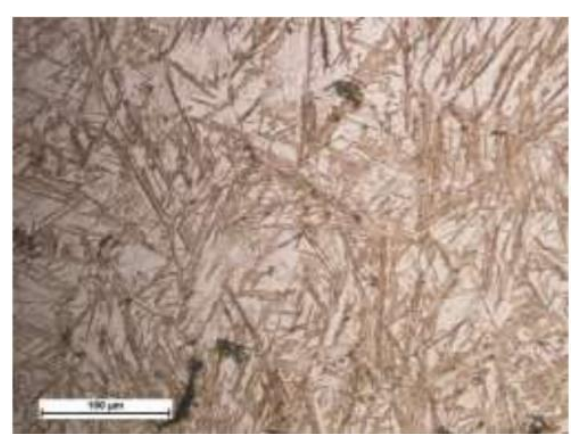

Fig. 2. Slag inclusions in padding weld [1]

Nowadays main on-line methods for slag inclusion detection are utilizing ultrasonic testing as well as eddy current NDT techniques [6,7]. Both methods demand contact (or near placement) of sensor and joint. Moreover tuning of measurement parameters is a nontrivial problem. More certain automatic methods are based on radiographic testing, that is connected with application of pattern recognition methods, like SVM classifiers [2] or more sophisticated machine learning methods, e.g. deep learning approaches [7,8]. Infrared thermography is rather unpopular technique for slag inclusion detection, in contrast to undercut or crack detection $[4,5]$.

A novel method of slag inclusion detection and localization in manual welding with coated electrode is proposed in the paper. It is based on on-line IR monitoring of joining process. Anomalies of temperature distribution described by set of complementary features are the fundament of diagnostic reasoning on the presence and location of slag inclusion. Proposed method can be applied for multi-pass welding to minimize number of low quality joints, that can be produced in electrode welding.

\section{Method of defects detection and localization}

Proposed method is based on observation of welding process with two LWIR cameras. Optical axis of first infrared camera is parallel to the direction of welding. This camera is used for observation of pool of molten metal an welding arc 
(Fig. 3a). Second camera is placed perpendicular to the direction of welding (Fig. 3b). It is used to observe the made joint in the cooling phase. Both cameras were uncooled microbolometer cameras, with resolution no lower than $640 \times 480 \mathrm{px}$, and the acquisition frequency was $50 \mathrm{~Hz}$.

For detection and localization two different methods were localized. Detection of slag inclusion was made with a global approach, connected with the drop of temperature during the cooling phase in small measurement areas placed equally on the entire seam.

The localization of inclusions was performed simultaneously assessing the dynamics of welding arc shape change, described by geometrical features of arc calculated for certain range of relative temperature increase. Additionally features connected the local speed of cooling in constant distance after the electrode tip was calculated. This type of calculation is needed because in manual welding there were slight variation of welding speed. One of most innovative approach was based on analysis of equation of a straight line that was fitted into part of heat profile in certain weld area describing the $t 8 / 5$ time (the cooling time between $800^{\circ} \mathrm{C}$ and $500^{\circ} \mathrm{C}$ ).

a)

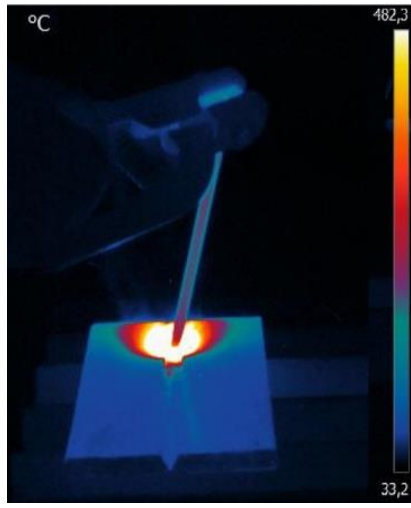

3. IR thermograms of welds b)

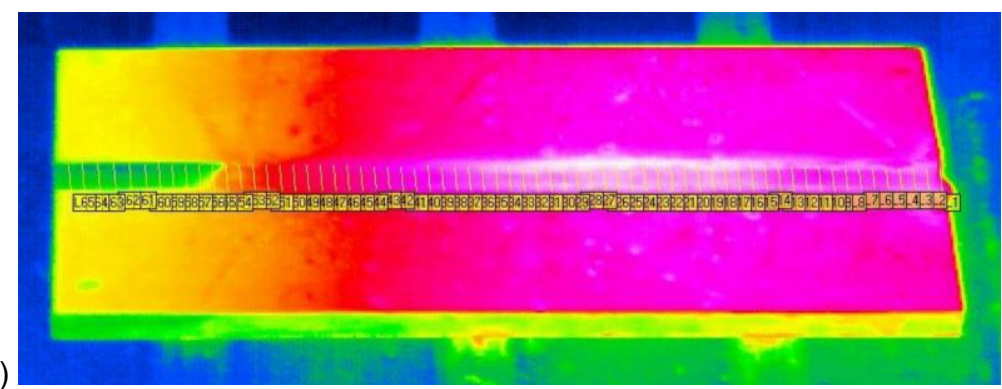

a) perpendicular to welding seam, b) parallel to welding seam, with marked measurement lines

\section{Results and conclusions}

Performed experiments led to manufacturing of set of welded joins, in which slag was intentionally included. Slag was laced in various localizations of cross section of joint consisting of two seams. the case of slag detection the first and the most important conclusion is that for samples without present imperfections temperature drop is slower, thus the mean temperature in areas that were cooled in same way is higher. This is connected with, the way that heat is dissipated by slag fraction. For slag localization differential signals were one of most promising ones, giving. Moreover processing of thermograms in frequency domain reduced unwanted noise. Comparison of IR based results with radiograms confirmed the propriety of proposed approach.

Future studies will cover investigations on possibility of distinguishing between inclusions and porosity. To achieve that a mechanical system connecting welder movements and camera translation will be proposed. Optimization of camera placement could influence the resolution of temperature distribution, increasing the possibility of smaller defects detection.

\section{REFERENCES}

[1] Żuk M., Górka J., Dojka R., Czupryński A., "Repair welding of cast iron coated electrodes“, IOP Conf. Ser.: Mater. Sci. Eng. 227 012139, 2017

[2] Shaohua Dong, Xuan Sun, Shuyi Xie, Mingfeng Wang, "Automatic defect identification technology of digital image of pipeline weld”, Natural Gas Industry B, vol. 6 (4), pp. 399-403, 2019

[3] Górka, J., Janicki, D., Fidali, M., Jamrozik W., "Thermographic Assessment of the HAZ Properties and Structure of Thermomechanically Treated Steel", Int J Thermophys vol. 38: 183, 2017

[4] Fidali M., Jamrozik W., "Diagnostic method of welding process based on fused infrared and vision images", Infrared Physics \& Technology, vol. 61, pp. 241-253, 2013

[5] Rodríguez-Martin M., Lagüela S., González-Aguilera D., Arias P., "Cooling analysis of welded materials for crack detection using infrared thermography”, Infrared Physics \& Technology, vol.67, pp. 547-554, 2014

[6] Lu Zhang, Amir Mostavi, Alexandra-Del-Carmen Basantes-Defaz, Didem Ozevin, J. Ernesto Indacochea, "The measurement of weld morphology and inclusions using ultrasonics", Measurement, vol. 144, pp. 33-43, 2019

[7] Rui Miao, Yuntian Gao, Liang Ge, Zihang Jiang, Jie Zhang, "Online defect recognition of narrow overlap weld based on two-stage recognition model combining continuous wavelet transform and convolutional neural network", Computers in Industry, vol. 112, 2019

[8] Liu, T.; Bao, J.; Wang, J.; Zhang, Y. A Hybrid CNN-LSTM Algorithm for Online Defect Recognition of CO2 Welding. Sensors, 18, 4369, 2018 